\title{
The Milky Way and the current status of galaxy formation models
}

\author{
Patricia B. Tissera \\ Institute for Astronomy and Space Physics, CC67 Suc 28, CABA 1248, Buenos Aires, \\ Argentina \\ email: ptissera@conicet.gov.ar
}

\begin{abstract}
I summarizes the main characteristics of a cosmological hydro-dynamical model which includes chemical evolution. In the last decade, galaxy formation models have made important progress which contribute to understand observations although there are still many open questions to be answered. Current chemo-dynamical cosmological simulations are able to reproduce global trends. They provide a suitable route to underpin physical events responsible of determining observed chemical patterns.
\end{abstract}

Keywords. galaxies:formation, galaxies:evolution, cosmology:theory.

\section{Introduction}

Precision cosmology favours a $\Lambda$-CDM Universe where baryons represent a small fraction of the total mass. This cosmological model has been so far successful at reproducing the properties of the matter in large and intermediate scales. How galaxies formed and evolved within dark matter haloes remains to be fully understood. It is at galaxy scales where baryons play a critical role and where many questions remain to be answered. Hydrodynamical cosmological simulations provide the best suitable tool to confront cosmology with observations. However, due to numerical limitations it is not possible to describe reliably all involved scales. Many processes occurring at galactic scales or smaller are modelled resorting to subgrid physics (which implies that only the effects taken place at resolved scales would be described). In the last decade, there has been enormous advances in the implementation of subgrid physics, principally related to Supernovae and radiative pressure feedback.

As stars evolve they synthesize chemical elements which are ejected into the ISM later on. The timescales and the detailed chemical production might imprint features in the stellar population and the gas component which can help us to understand how their parent galaxies formed. In order to do that, chemical evolution has been included into hydrodynamical cosmological codes. Chemical patterns might store fossil records of the physical characteristics of the interstellar medium at the time and place of their birth and the physical processes which affect them (see Bland-Hawthorn \& Freeman 2003 for a detail review). We will be able to explore this if we can link chemical patterns with particular events in galaxy formation. It is also worth mentioning that to confront models and observations we need to improve the way these comparisons are carried out.

Recent observations provide evidence that the Milky Way halo system comprises at least two overlapping stellar components, an inner-halo population and an outer-halo population, with different metallicities, kinematics, and spatial density profiles (see e.g., Carollo et al. 2007). Forthcoming instruments and survey will provide an enormous amount of data which will pose a challenge to galaxy formation. Galaxy formation models which 
include chemical evolution are a powerful route to understand observations within a cosmological model.

In this proceeding, I summarizes our chemo-dynamical cosmological models and some of the results obtained for Milky Way mass-sized galaxies.

\section{Numerical Simulations}

We analyze a suite of Milky Way mass-size galaxies from the Aquarius Project which have been resimulated by including baryons (Scannapieco et al. 2009). The target haloes were selected from a cosmological volume consistent with a $\Lambda$-CDM cosmogony, with

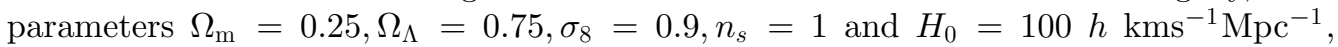
and $h=0.73$. The simulations were run with a version of GADGET-3 (Springel 2005), which includes a multiphase medium, SN energy feedback, and chemical evolution, as described in Scannapieco et al. $(2005,2006)$. The simulated haloes have $\approx 1$ million total particles within the virial radius, and a virial mass in the range $\approx 5-11 \times 10^{11} \mathrm{M}_{\odot} h^{-1}$ at $z=0$. Their characteristic parameters are summarized in table 1 and table 2 of Scannapieco et al. (2009). The SN feedback model considers SNII and SNIa energy and chemical productions. The metals produced by SN events by a given stellar particle is distributed within the closest neighbours, weighting following Mosconi et al. (2001). SNII are assumed to be produced by stars more massive than $8 M_{\odot}$ and chemical yields are taken from Wooley \& Weaver (1995). SNIa are estimated by assuming an observational motivated rate and the yields are taken from W7 model of Thielmann et al. (1993). A Salpeter Initial Mass Function has been adopted.

The SN energy is distributed differently according to the specific entropy of the gas surrounding the star particle. A fraction $\epsilon_{c}$ of the SN energy is injected into the low entropy gas neighbours while the rest is pumped in the high entropy ones (the reference entropy value has been set and discussed in Scannapieco et al. (2006)). A low entropy gas particle stores this energy in a reservoir until enough has been accumulated to increase the entropy to levels comparable to its hot neighbours. This SN feedback model has proven to be efficient at driving mass-loaded galactic winds which are able to carry out metals outside galaxies. The strength of the outflows depends on the potential well of the systems as shown in Scannapieco et al. (2006).

\section{Global chemical properties of stars in different dynamical components}

Our numerical model allows us to follow the formation of the structure while describing the chemical enrichment of baryons. So, as galaxies are assembled, we can study the chemical properties of their different dynamical components. For that purpose, we defined disc, bulge, inner and outer haloes components (hereafter, inner halo populations (IHPs) and outer haloes populations (OHPs). Stars in the disc components have been selected as those that are rotational supported. The remaining stars are part of the spheroidal component and are separated in bulge, IHP and OHP, by adopting criteria based on the binding energy of the star (see Tissera et al. (2012) for details on the component definitions).

When stars have been sorted out in the four dynamical components, we compare their chemical properties. Globally, our simulations are able to describe general properties of observed Spiral galaxies: the bulges show the higher levels of $[\mathrm{Fe} / \mathrm{H}]$ and are $\alpha$-enriched while the inner haloes and outer haloes have low $[\mathrm{Fe} / \mathrm{H}]$ and high $\alpha$-enriched. Stars in the discs tend to be low $\alpha$ and have high metallicity. In Fig. 1, we show the cumulative stellar 

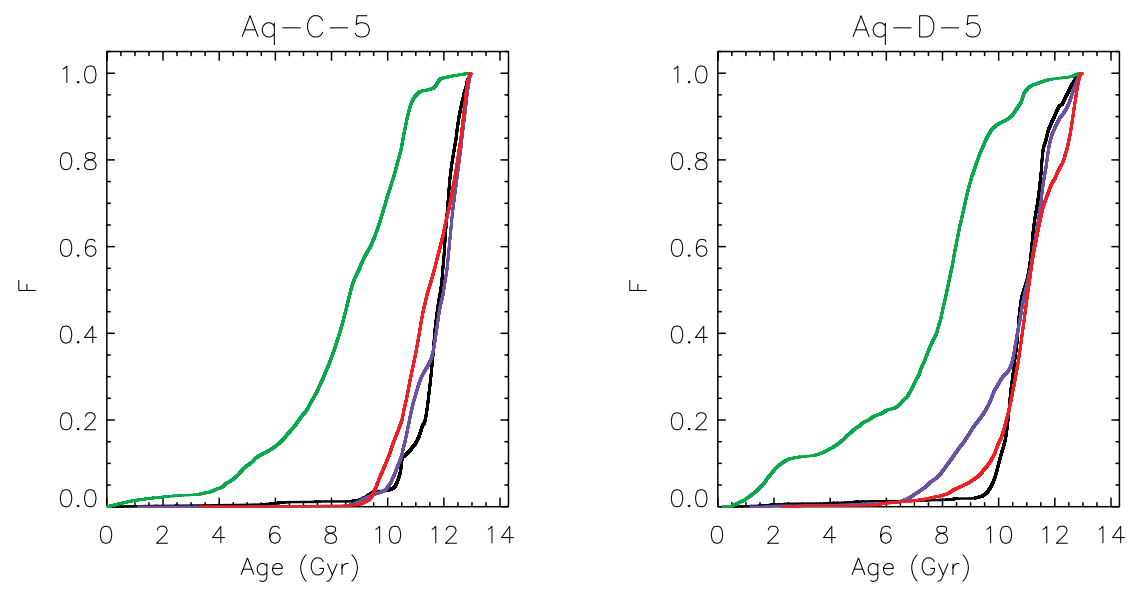

Figure 1. Cumulative stellar mass as a function of stellar age for the disc (green lines), bulge (black lines), inner (violet lines) and outer (red lines) components in two Aquarius systems. Stars in the bulge and halo components are comparably old, but their chemical properties are different. Stars in the bulge tend to have higher metallicities than those in the haloes while both are $\alpha$-enriched, on average. The differences in the metallicity levels reflect the different history of formation: stars in bulges formed in deeper potential wells (The light gray lines represent the disc component and dark lines the three spheroidal subcomponents (see Tissera et al. (2012)).

mass as a function of age of stars in the four components for two Aquarius galaxies. We can see that the discs are formed by younger stars than the rest of the components which have formed during an extended time period. Conversely, stars in the bulges, IHPs and OHPs are old. Nevertheless, the chemical abundances of stars in the bulge and haloes are different reflecting their very different formation histories. Most of stars in the bulges formed in the deep potential wells while those which formed in the stellar haloes tend to form in smaller satellites.

The analysis has been done for stars in the stellar haloes where the surviving satellites have been taken out. However, the streams remain as can be seen from $j_{z} v s E$ plane in figure 1 of Tissera et al. (2012). In Fig. 2 we show the projected baryonic distribution of one of our systems where it can be appreciated the surviving satellites embedded within the underlying stellar halo as well as the presence of streams.

We followed each stellar particle in time and identified where they formed. In general terms, we classify as insitu those stars that formed within the virial radius of the main progenitor at any epoch. If they formed in separate satellites outside the main galaxy at any epoch, we classify them as part of the debris subpopulations.

We also investigated in more details where the insitu stars originated: if they formed in separated satellites, but within the virial radius, we named them 'endo-debris' subpopulation and if they formed in a disc component they are taken as disc-heated subpopulation. The endo-debris subcomponents have been defined because these stars exhibit different chemical properties than debris stars. Endo-debris stars tend to less $\alpha$-enriched evidencing enrichment by SNIa.

According to this classifications, most of stars in the bulge and in the disc components are formed insitu with a small fraction of debris stars. OHPs are mainly formed by debris stars with less than $20 \%$ coming from the endo-debris subcomponent. IHPs are formed by comparable contributions from debris and insitu stars, depending on the systems. And on its turn, the insitu subcomponents are a mixture of endo-debris $(\approx 30 \%)$ and disc-heated (from $0 \%$ to $30 \%$ ) stars. The fractions of disc-heated stars should be taken as 


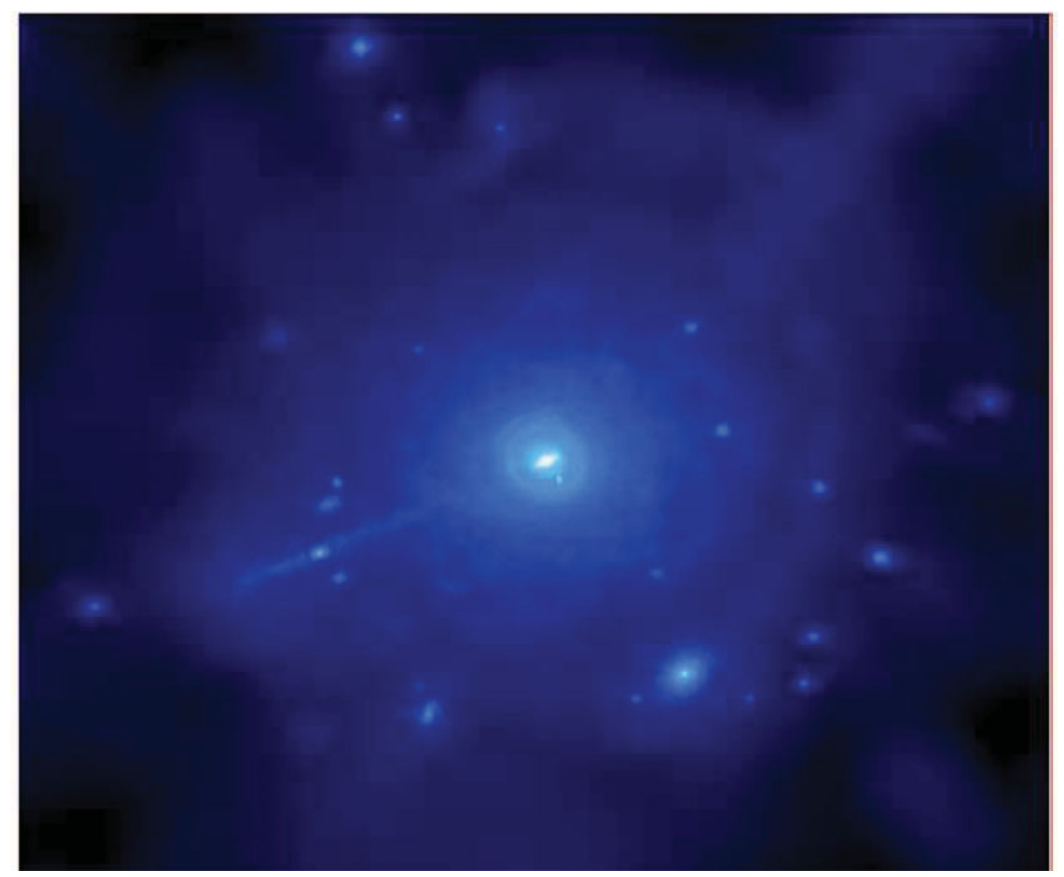

Figure 2. Baryons distributions in one our simulated Aquarius haloes. We can see the main galaxy with its disc, the surviving satellites, the extended stellar haloes and remaining streams.

upper limits since there are several numerical uncertainties which can artificially increase their contributions. The $[\mathrm{O} / \mathrm{Fe}]$ versus $[\mathrm{Fe} / \mathrm{H}]$ distributions of our simulated IHPs agree very well with recent observational results reported by Sheffield et al. (2012).

Both the IHPs and OHPs show metallicity gradients. The metallicity profiles of the IHPs are determined by the combination of insitu and debris stars while those of the OHPs are mainly affected by the mass of the accreted satellites. Those OHPs formed by a larger contribution of stars originated in massive satellites would tend to show a steeper metallicity profile (see also Cooper et al. (2010)). The combination of both the IHPs and OHPs lead to global abundance distribution which might vary from the inner to the outer part of the stellar haloes (see Fig. 3). The characteristics of these variations store information on the way they stellar haloes were assembled (see Tissera et al. in preparation).

Regarding the kinematics, we found that most of the OHPs exhibit low net global rotation with respect to their corresponding galactic centres. We found mean rotational velocity values around $20-25 \mathrm{~km} \mathrm{~s}^{-1}$. One of our haloes exhibits a very low net rotation of $\sim 7 \mathrm{~km} \mathrm{~s}^{-1}$ while another one shows retrograde net rotation of $-33 \mathrm{~km} \mathrm{~s}^{-1}$.

\section{Final Remarks}

We show that state-of-art numerical simulations which include chemical evolution are a powerful tool to understand observations within galaxy formation models. The confrontation of the chemo-dynamical properties of simulated and observed galaxies also provides a suitable route to improve sub-grid physics. Current galaxy formation models can reproduce observed global metallicity trends. Regarding the stellar haloes, numerical results show that $\Lambda$-CDM could explain their global properties. However, a detailed and robust comparison needs improvement in both the models and how models are compared 

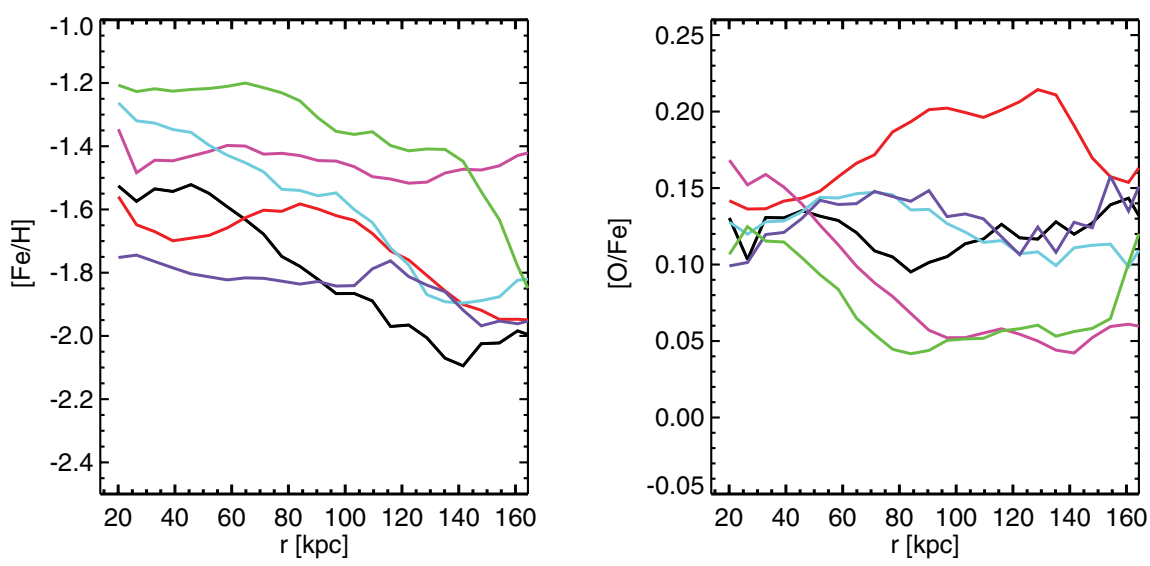

Figure 3. Median abundance profiles of $[\mathrm{Fe} / \mathrm{H}]$ for stars in the haloes located from $20 \mathrm{kpc}$ up to the virial radius. There is a variety of behaviours, from flat to steeper gradients. There is a trend for haloes formed from a large contribution from massive satellites to have steeper (and more enriched, on average) abundance profiles than those formed from more alike ones. We find to clear trend for $[\mathrm{O} / \mathrm{Fe}]$ which seems to be more sensitive to the presence of substructures such as streams. Different (colour) lines represent each analyzed Aquarius Haloes (see Tissera et al. (2013).

to observations. Forthcoming large surveys of the Milky-Way such as Gaia and LaMOST, and observations of stellar haloes in other galaxies would contribute towards these aims.

\section{Acknowledgements}

PBT thanks the LOC and SOC for the invitation to give this talk and for their help. PBT acknowledge the IAU for providing partial support to attend the Symposium. This work was partially funded by a local L'Oreal-Unesco Award for Women in Science and Cosmocomp and Lacegal Networks of FP7 Programme of the European Community.

\section{References}

Bland-Hawthorn, J. \& Freeman, K. C. 2003, in: Perez, E., Gonzalez Delgado, R. M., TenorioTagle, G., eds., Star Formation Through Time, ASP, San Francisco, p. 457

Carollo, D., et al. 2007, Nature, 450, 1020

Cooper, A. P., et al. 2010, MNRAS, 450, 1020

Mosconi, M., Tissera, P. B., Lambas, D. G. \& Cora, S. A. 2001, MNRAS, 325, 34

Scannapieco, C., Tissera, P. B., White, S. D.M. \& Springel, V. 2005, MNRAS, 364, 552

Scannapieco, C., Tissera, P. B., White, S. D.M. \& Springel, V. 2006, MNRAS, 371, 1125

Scannapieco, C., White, S. D.M., Springel, V. \& Tissera, P. B. 2009, MNRAS, 396, 696

Sheffield, A. A., et al. 2012, ApJ, 761, 161

Springel, V. 2005, MNRAS, 364, 1105

Tissera, P. B., Scannapieco, C. \& White, S. D.M. 2012, MNRAS, 420, 255

Tissera, P. B., Scannapieco, C., Beers, T. \& Carollo, D. 2013, MNRAS, 432, 3391

Thielemann, F., Nomoto, K., Hashimoto, M. 1993, Origin and Evolution of the Elements, Cambridge Univ. Press, p. 297

Woosley, S. E. \& Weaver, T. A. 1995, ApJS, 101, 181 


\section{Discussion}

MASSEY: I am wondering if you have considered the expected intrinsic dispersion in absolute magnitude of WRs? if you consider the (large) mass range that becomes an early WN or late WC according to the evolutionary models, wouldn't you expect a large dispersion in $\mathrm{M}_{V}$ ?

TISSERA: Indeed, we will be always left with some intrinsic scatter in $\mathrm{M}_{V}$ due to mass differences within the same spectral subtype. But in my opinion, the current large dispersion is for a large fraction due to uncertainties of the adopted distances of open clusters and $\mathrm{OB}$ associations. 